\title{
Advances in Stellarator Gyrokinetics
}

\author{
P. Helander, T. Bird, F. Jenko, R. Kleiber, G.G. Plunk, J.H.E. Proll, J. Riemann, P.
} Xanthopoulos

Max-Planck-Institut für Plasmaphysik, Greifswald and Garching, Germany

Recent progress in the gyrokinetic theory of stellarator microinstabilities and turbulence simulations is summarised. The simulations have been carried out using two different gyrokinetic codes, the global particle-in-cell code EUTERPE and the continuum code GENE, which operates in the geometry of a flux tube or a flux surface but is local in the radial direction. Ion-temperature-gradient (ITG) and trapped-electron modes are studied and compared with their counterparts in axisymmetric tokamak geometry. Several interesting differences emerge. Because of the more complicated structure of the magnetic field, the fluctuations are much less evenly distributed over each flux surface in stellarators than in tokamaks. Instead of covering the entire outboard side of the torus, ITG turbulence is localised to narrow bands along the magnetic field in regions of unfavourable curvature, and the resulting transport depends on the normalised gyroradius $\rho_{*}$ even in radially local simulations. Trapped-electron modes can be significantly more stable than in typical tokamaks, because of the spatial separation of regions with trapped particles from those with bad magnetic curvature. Preliminary nonlinear simulations in flux-tube geometry suggest differences in the turbulence levels in Wendelstein 7-X and a typical tokamak. 


\section{Introduction}

Wendelstein 7-X will start operation in 2015 and should relatively quickly be able to demonstrate how well the magnetic field has been optimised with respect to neoclassical transport, but the more the latter is suppressed, the more vulnerable the confinement becomes to turbulence [1]. Since the neoclassical heat flux scales strongly with temperature (as $T^{9 / 2}$ in the typical $1 / \nu$-regime for electrons), it is expected to dominate over turbulent losses in the centre of high-temperature discharges. However, turbulent tranport is expected to be important in at least the outer half of the plasma volume, as consistently observed in the predecessor experiment, Wendelstein 7-AS [2]. It is therefore of great interest to understand and predict the nature and the level of the turbulence expected in W7-X.

The present article summarises a number of recent advances made in the understanding of gyrokinetic microinstabilities and turbulence simulations in stellarator geometry. Since the general understanding of turbulence in stellarators is still rudimentary, we restrict our attention to the simplest collisionless and electrostatic instabilities, but the underlying key question is one of broad interest in plasma physics: how does the magnetic-field geometry affect plasma stability and turbulence?

We have addressed this question using analytical theory of linear microinstabilities and gyrokinetic simulations in non-axisymmetric three-dimensional magnetic geometry, using two gyrokinetic stellarator codes under development for this purpose. The first code is EUTERPE, a global, electromagnetic particle-in-cell code, which has been used to calculate linear growth rates with and without electromagnetic effects taken into account [3]. The other code is the stellarator version of GENE $[4,5]$, which has been extended so that it can simulate turbulence not only in a flux tube but on an entire magnetic surface whilst still employing a local approximation in the radial direction [6]. Thus, the metric elements vary in the poloidal and toroidal directions, but are constant in the radial direction, and the fluctuations obey periodic boundary conditions in all these three directions. The GENE code is thus global in the angular directions but local in the radial direction, whereas EUTERPE is fully global. On the other hand, GENE is nonlinear and EUTERPE linear when running in stellarator geometry. These two

codes make it possible to compare the properties of microinstabilities and turbulence in stellarators and tokamaks. 


\section{The ITG mode}

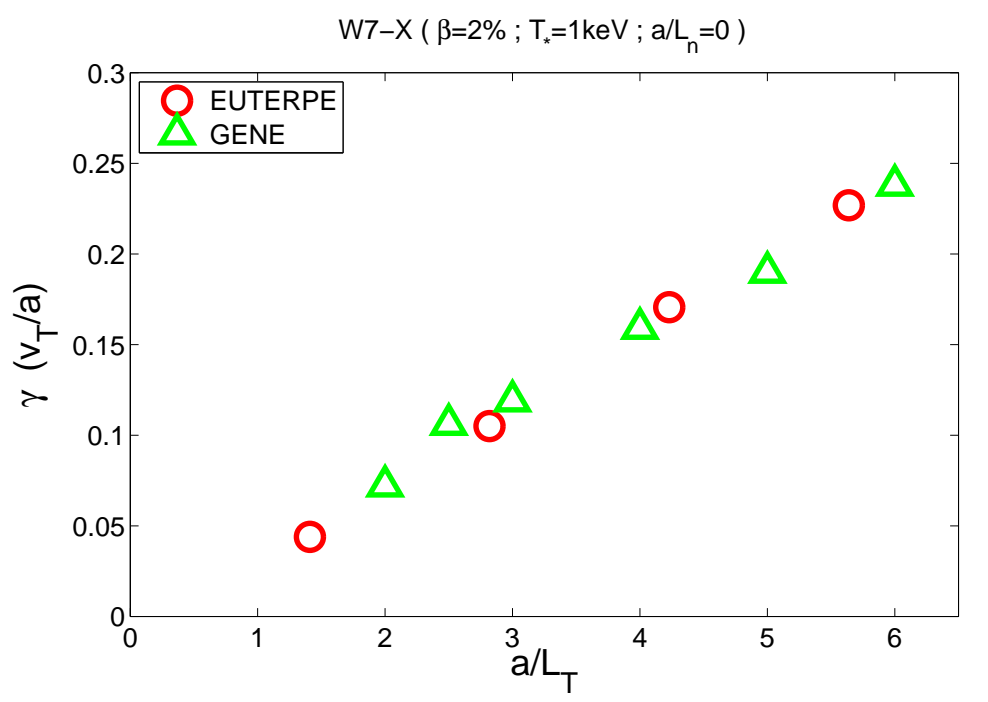

Figure 1: ITG instability growth rate in W7-X with Boltzmann electrons computed by the EUTERPE and GENE codes. The growth rate is shown in units of $v_{T i} / a=$ $\left(T_{i} / m_{i}\right)^{1 / 2} / a$, and the ion temperature on the $s=1 / 2$ surface is equal to $T_{i}=1 \mathrm{keV}$.

In tokamaks, the two most important electrostatic microinstabilities are the iontemperature-gradient (ITG) mode and the trapped-electron mode (TEM). These are predicted to be present in stellarators, too, but their properties can be different [7, $8,9,10,11,12,13]$. The curvature-driven ITG mode is affected by the fact that the temperature gradient is very large in regions where the magnetic surfaces are tightly spaced, e.g., in the "corners" (the bean-shaped cross sections) of W7-X, where the plasma cross section is bean-shaped and highly elongated. On the other hand, the connection length along the magnetic field between regions of good and bad curvature is significantly shorter than in a typical tokamak, which is stabilising for the toroidal branch of the ITG mode. Figure 1 shows a comparison of the growth rates found in linear simulations of the ITG mode assuming Boltzmann-distributed electrons using EUTERPE and GENE, respectively. (This comparison was carried out as a "blind test", where the participants did not exchange information during the course of the simulations.) The magnetic geometry is that of the "high-mirror" configuration of W7-X [14], the density gradient is taken to vanish, and the ion temperature gradient 
employed in EUTERPE is given by the piecewise linear function

$$
\frac{d \ln T}{d s}=-\frac{a \sqrt{2}}{L_{T}}\left(\frac{1}{2}-\left|s-\frac{1}{2}\right|\right),
$$

where $s=\psi / \psi_{a}$ denotes the toroidal flux normalised to its value at the plasma edge, whose average minor radius is $a=0.55 \mathrm{~m}$. It is observed in the EUTERPE simulations that the eigenmode peaks around the surface $s=1 / 2$, where $d \ln T / d s$ has its maximum, and this is the radial location chosen for the GENE simulations. The mode structures calculated by the two codes on this surface are also very similar. Figure 2 shows the distribution of potential fluctuations over the $s=1 / 2$ surface calculated by EUTERPE. The instability peaks close to (but not exactly in) the outboard midplane in the beanshaped cross section of the device, where the curvature is particularly unfavourable.

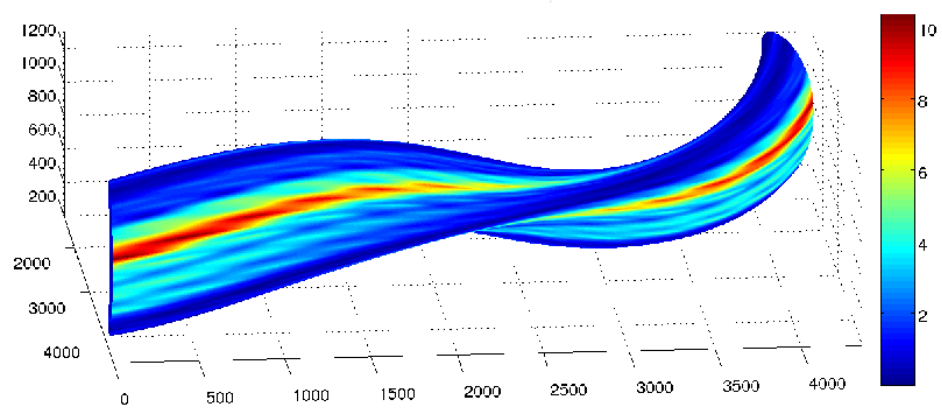

Figure 2: Linear mode structure of an ITG mode on the $s=1 / 2$ magnetic surface in W7-X. The absolute value of the electrostatic potential is plotted (in arbitrary units since the simulation is linear), and the lengths used on the axes are expressed in units of ion gyroradii.

EUTERPE simulations of linear ITG modes in W7-X and LHD geometry indicate that the growth rate is almost identical in these two devices although the mode structure is affected by details in the magnetic geometry and therefore different. The growth rate is shown in Fig. 3 as a function of the normalised temperature gradient $a / L_{T}$ and its ratio to the density gradient, $\eta=(d \ln T / d s) /(d \ln n / d s)$. Here and in the following, $L_{T}$ is defined by

$$
\frac{d \ln T}{d r}=\frac{1}{L_{T}}
$$

in terms of the radial coordinate $r=a \sqrt{s}$. As usual in simulations without kinetic electrons, there is no linear instability below a threshold at $\eta \simeq 1$. Electromagnetic 

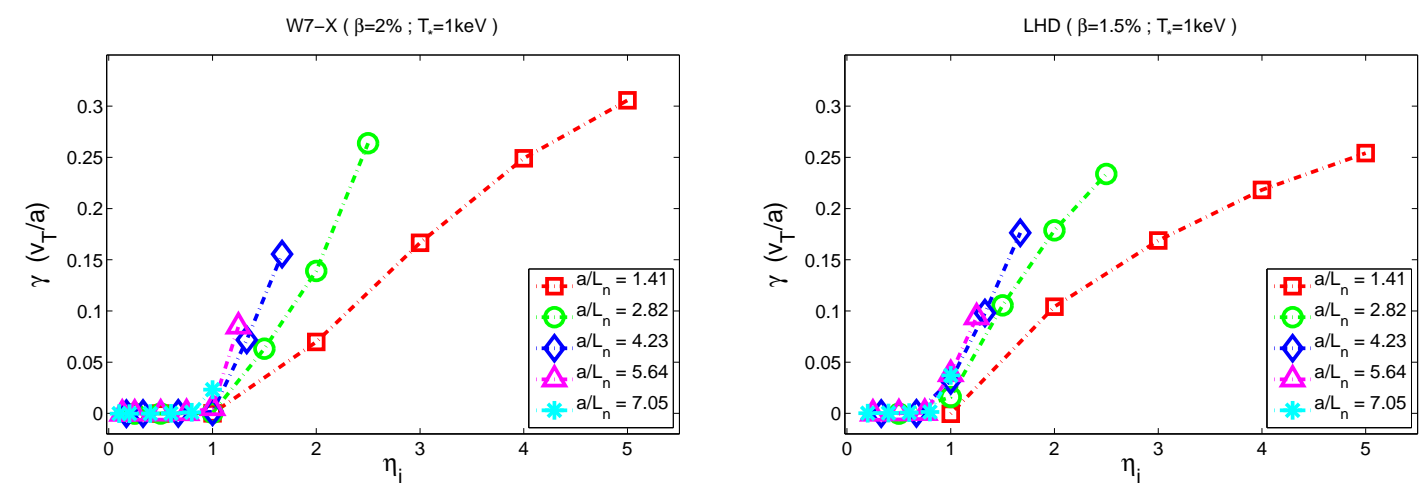

Figure 3: ITG instability growth rate in W7-X and LHD as functions of $\eta=d \ln T / d \ln n$ and $a / L_{T}$ on the surface $s=1 / 2$, where $T=1 \mathrm{keV}$, calculated with the EUTERPE code. The growth rate is shown in units of $v_{T} / a$, where $v_{T}$ is the ion thermal speed and $a$ the minor radius.

effects appear to be modest in all cases investigated so far, and no clear evidence of kinetic ballooning modes has been encountered.

Nonlinear GENE simulations of saturated ITG turbulence in W7-X with Boltzmanndistributed electrons show that the turbulent density fluctuations, too, are much less evenly distributed over the flux surface than in typical tokamak simulations $[6,10]$. Instead of covering the entire outboard side of the torus, the turbulence is localised to narrow bands in regions of bad magnetic curvature, see Fig. 4, much like the linear mode structure in Fig. 2. Another difference to typical tokamak simulations is that the range of fluctuation amplitudes is much greater. 


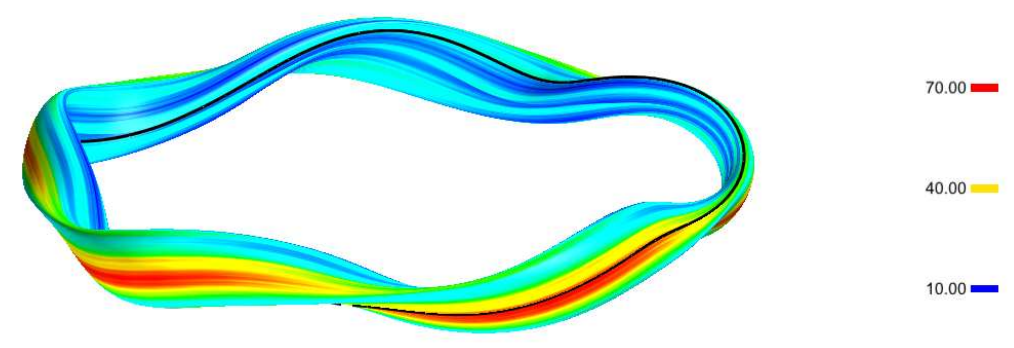

Figure 4: Root-mean-squared potential fluctuations in units of $T_{i} / e \rho_{*}$ in a GENE simulation of ITG turbulence on a flux surface of Wendelstein 7-X with $a / L_{T}=2.5$ and $\rho_{*}=1 / 125$.

Probably as a consequence of the poloidal localisation of the turbulence, the transport scaling is sensitive to the normalised gyroradius, $\rho_{*}=\rho_{i} / a$, and becomes "stiffer" when $\rho_{*}$ is reduced, see Fig. 5 [6]. Flux-tube simulations correspond to the limit $\rho_{*} \rightarrow 0$, in which the gyroradius scale decouples from the macroscopic scale, and one would perhaps expect that full-surface simulations should approach flux-tube ones when $\rho_{*}$ is made very small. It appears that this is indeed the case in the sense that the transport observed in full-surface simulations never exceeds that in simulations of the most unstable flux tube. In stellarators, different flux tubes (of finite length) on the same magnetic surface are not equivalent to each other but exhibit different growth rates in linear simulations as well as different turbulent transport levels in nonlinear ones. In W7-X, the most unstable flux tube is the one that passes through the horizontal midplane in the bean-shaped cross section, and this is the tube chosen for all nonlinear simulations presented in this paper. The transport across this tube is shown by inverted triangles in Fig. 5 and always lies above the full-surface results, with the difference diminishing as $\rho_{*} \rightarrow 0$. We speculate that in this limit the transport across the full surface approaches the corresponding average of all flux tubes within the surface. Note that the transport is different from that in tokamaks, where flux-tube simulations usually yield the same results as simulations of entire flux surfaces. In the stellarator, there is thus a "nonlocality" of the turbulence within the flux surface, in addition to any radially nonlocal effects. Full-volume turbulence simulations have not yet been 
performed in stellarator geometry, but it is well known that the transport in global tokamak simulations can depend on $\rho_{*}$ due to radially non-local effects [15], and there is no reason to believe that this should not be the case in stellarators, too.

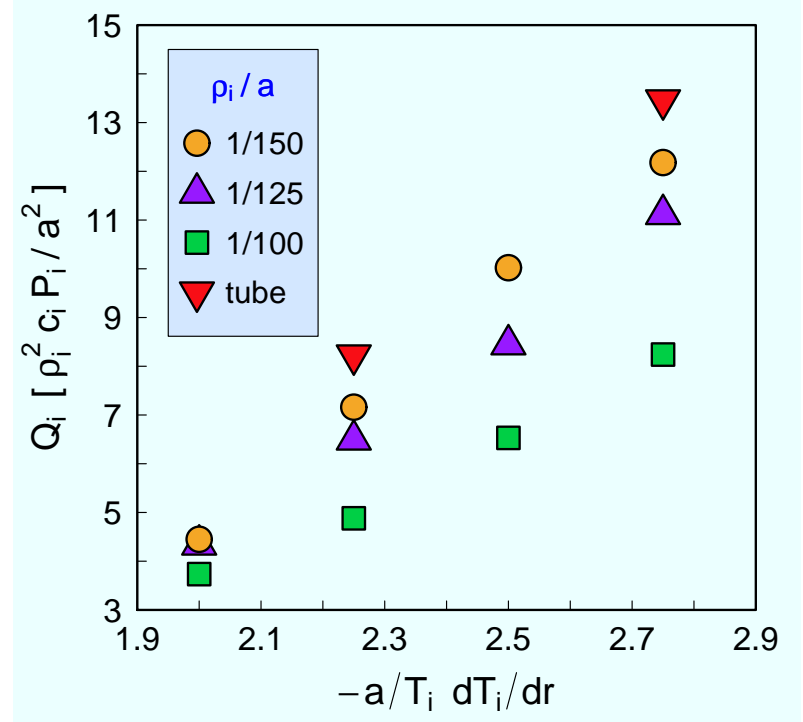

Figure 5: The heat flux vs temperature gradient for three different values of the normalised ion gyroradius $\rho_{*}=\rho_{i} / a$ in radially local ITG turbulence simulations using GENE with Boltzmann-distributed electrons in W7-X. Results from flux-tube simulations are displayed for comparison.

In order to understand why the turbulence is likely to be affected by the fact the poloidal width of the unstable region is small, we recall the simplest local dispersion relation for curvature-driven ITG modes, as found, e.g., in Eq. (12) of Ref. [13],

$$
\tau \omega^{2}+(1-b \eta) \omega_{* i} \omega+\eta \omega_{d i} \omega_{* i}=0
$$

where $\omega_{* i}=\left(T_{i} k_{\alpha} / e\right) d \ln n / d \psi$ denotes the diamagnetic frequency, $\omega_{d i}=\mathbf{k}_{\perp} \cdot \mathbf{v}_{d i}$ the ion drift frequency, $\tau=T_{i} / T_{e}$ the ion-to-electron temperature ratio, and $b=k_{\perp}^{2} T_{i} / m_{i} \Omega_{i}^{2}=$ $k_{\perp}^{2} \rho_{i}^{2}$. The wave vector has been written as $\mathbf{k}_{\perp}=k_{\psi} \nabla \psi+k_{\alpha} \nabla \alpha$ and the magnetic field as $\mathbf{B}=\nabla \psi \times \nabla \alpha$. The simulations of Fig. 5 were carried out with a temperature gradient but no density gradient, so that $\omega_{* i 0}=0$ but $\eta \omega_{* i 0}=\omega_{T} \neq 0$, and since $\tau=1$ the real part of the frequency thus becomes $\omega_{r}=b \omega_{T} / 2$. The group velocity at which 
turbulent fluctuations with $k_{\alpha} \neq 0$ propagate in the poloidal $(\alpha$-) direction is thus

$$
v_{\alpha}=\frac{\partial \omega_{r}}{\partial k_{\alpha}}=\frac{3 b \omega_{T}}{2 k_{\alpha}}
$$

In order for conventional instability-driven plasma turbulence to arise, small fluctuations must have time to grow and saturate nonlinearly before they are convected out of the bad-curvature region. If the poloidal width of this region is $\Delta \alpha$, the time that a fluctuation spends there is $\Delta t=\Delta \alpha / v_{\alpha}$, and during this time it experiences a growth of order

$$
\exp (\gamma \Delta t)=\exp \left(\frac{2 k_{\alpha} \Delta \alpha}{3 b} \frac{\gamma}{\omega_{T}}\right)
$$

Clearly, if $\Delta \alpha$ is sufficiently small, this growth factor is not very large and a linear fluctuation leaves the unstable region before it has experienced much growth. Moreover, this happens even if $\Delta \alpha$ is not very small (i.e., even if this width corresponds to many ion gyroradii) since the ratio $\gamma / \omega_{T}$ is considerably smaller than unity according to Fig. 3, because $\omega_{T} \sim k_{\perp} \rho_{i} v_{T} / L_{T}$ where $1 / 2<k_{\perp} \rho_{i}<1$ for the fastest growing mode. In any case, under such circumstances one would expect the turbulence to "notice" the finite width of the unstable region it lives in, rendering flux-tube simulations insufficient to faithfully represent the true behaviour of the system. (In principle this could happen in a tokamak, too. The important point is that the macroscopic length scale set by the poloidal variation of the drift frequency $\omega_{d i}$ may be shorter than that customarily used in the definition of $\rho_{*}$.) A more detailed analysis of this phenomenon, including the derivation of poloidally non-local dispersion relations, will be published separately.

\section{Trapped-electron modes}

Trapped-electron modes can be very different in tokamaks and stellarators, because these instabilities are excited by trapped electrons in regions of unfavourable magnetic curvature. Tokamaks and stellarators are fundamentally different in the sense that the regions of trapping and bad curvature overlap in tokamaks - both being on the outboard side of the torus - whereas in stellarators they can be located in different places. In particular, quasi-isodynamic stellarators can have the property that the bounce-averaged curvature is favourable for the great majority of all trapped particles. 


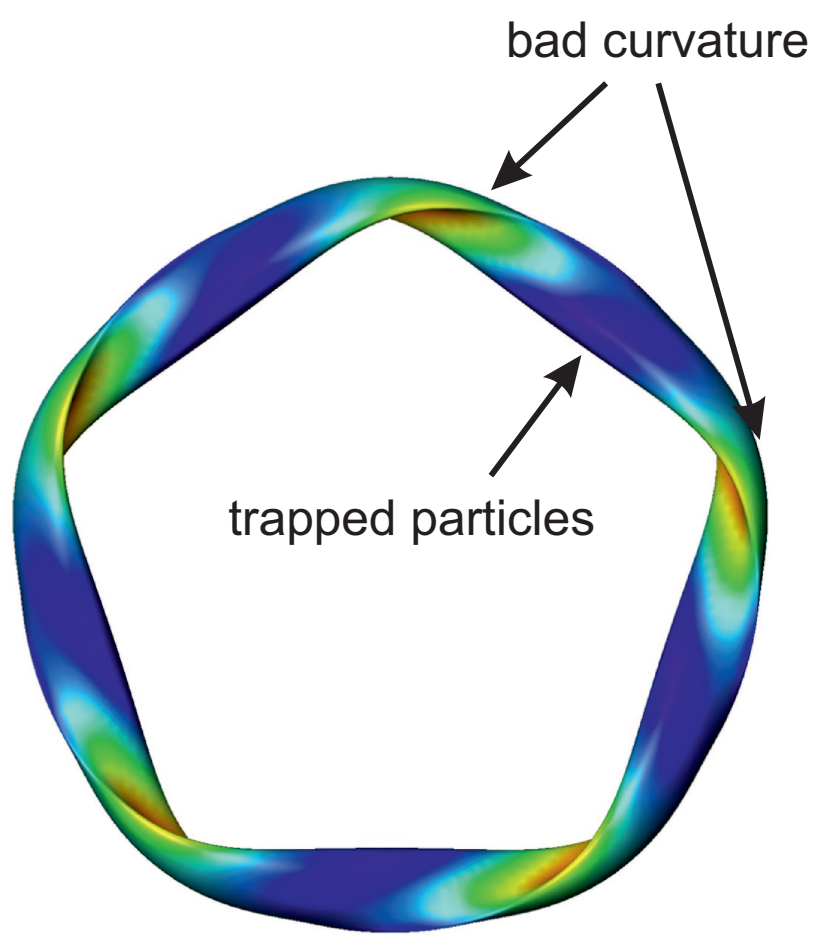

Figure 6: In W7-X, the magnetic-field strength is largest in the "corners" of the device (the bean-shaped cross sections), where the curvature is particularly large and unfavourable. Therefore, the regions of magnetic trapping and bad curvature do not overlap to the same extent as in a tokamak.

In a quasi-isodynamic stellarator, the parallel adiabatic invariant, defined as

$$
J(\psi, \alpha, \lambda)=m_{a} v \int \sqrt{1-\lambda B(l)} d l,
$$

taken between two consecutive bounce points, where $\lambda B=1$, is independent of $\alpha$ [16]. Here $v$ denotes the speed and $m_{a}$ the mass. On a bounce average (denoted by an overbar), the magnetic drift is equal to

$$
\overline{\mathbf{v}_{d a} \cdot \nabla \psi}=\frac{1}{e_{a} \tau_{b a}} \frac{\partial J}{\partial \alpha}, \quad \overline{\mathbf{v}_{d a} \cdot \nabla \alpha}=-\frac{1}{e_{a} \tau_{b a}} \frac{\partial J}{\partial \psi},
$$

where $e_{a}$ is the charge and $\tau_{b a}$ the bounce time of species $a$. The sign of $\partial J / \partial \psi$ thus governs the direction of the poloidal precession, which is opposite to the diamagnetic flow if $\partial J / \partial \psi$ is negative. In such, so-called maximum- $J$ configurations [17], it can be shown analytically that collisionless TEMs are stable in large parts of parameter space 
$[11,18]$. The physical reason is that the interaction between the electrons and any instability with a frequency far below the electron bounce frequency has to conserve $J$ for all participating electrons [10]. Suppose that such an instability taps into the free energy of the density gradient, $d n / d r<0$, by moving an electron radially a small distance $\Delta r>0$. The change in $J$ is then

$$
\Delta J=\frac{\partial J}{\partial r} \Delta r+\frac{\partial J}{\partial E} \Delta E=0
$$

where $E=m_{e} v^{2} / 2$, and the electron thus gains the energy

$$
\Delta E=-\frac{\partial J / \partial r}{\partial J / \partial E} \Delta r
$$

at the expense of the instability. Since $\partial J / \partial E>0$, it follows that the condition $\partial J / \partial r<0$ promotes stability.

This argument can be made precise by considering the energy budget of the gyrokinetic equation $[11,18]$.

$$
i v_{\|} \nabla_{\|} g_{a}+\left(\omega-\omega_{d a}\right) g_{a}=\frac{e_{a} \phi}{T_{a}} J_{0}\left(\frac{k_{\perp} v_{\perp}}{\Omega_{a}}\right)\left(\omega-\omega_{* a}^{T}\right) f_{a 0},
$$

for the distribution function $f_{a}=f_{a 0}+f_{a 1}+\cdots$. Here, its nonadiabatic component is denoted by $g_{a}=f_{a 1}+\left(e_{a} \phi / T_{a}\right) f_{a 0}$, and we have written

$$
\omega_{* e}^{T}=-\frac{T_{e} k_{\alpha}}{e} \frac{d \ln n}{d \psi}\left[1+\eta_{e}\left(\frac{m_{e} v^{2}}{2 T_{e}}-\frac{3}{2}\right)\right],
$$

with $\eta_{e}=d \ln T_{e} / d \ln n_{e}$. The work done by the electric field of an electrostatic instability, $\mathbf{E}=-\nabla \phi$, on the guiding centers of an arbitrary particle species $a$ is

$$
P_{a}=-e_{a}\left\{f_{a 1}\left(v_{\|} \mathbf{b}+\mathbf{v}_{d a}\right) \cdot \nabla \phi\right\}=e_{a}\left\{\phi\left(v_{\|} \mathbf{b}+\mathbf{v}_{d a}\right) \cdot \nabla f_{a 1}\right\}
$$

where $\mathbf{b}=\mathbf{B} / B$ and we have introduced the notation

$$
\{\cdots\}=\int_{-\infty}^{\infty} \frac{d l}{B} \int(\cdots) d^{3} v
$$

for an integral over velocity space and along the entire field line in ballooning space. In the usual drift-wave approximation, $\omega \ll k_{\|} v_{T e}$, where $v_{T e}$ denotes the electron thermal speed, the linear gyrokinetic equation can be solved analytically,

$$
g_{e 0}^{\mathrm{tr}}=-\frac{\omega-\omega_{* e}^{T}}{\omega-\bar{\omega}_{d e}} \frac{e \bar{\phi}}{T_{e}} f_{e o},
$$


where overbars again denote bounce averages. The transfer rate of electrostatic energy from the instability to the electron population is thus found to be given by $[13,18]$

$$
P_{e}=-\frac{e^{2}}{T_{e}}\left\{|\phi|^{2} \frac{\gamma}{\left.\left(\omega_{r}-\bar{\omega}_{d e}\right)\right)^{2}+\gamma^{2}} \bar{\omega}_{d e}\left(\omega_{* e}^{T}-\bar{\omega}_{d e}\right) f_{e 0}\right\} .
$$

It is evident from this result that $P_{e}>0$ if the product

$$
\omega_{* e} \bar{\omega}_{d e}=-\frac{k_{\alpha}^{2} T_{e}}{e^{2} \tau_{b e}} \frac{d \ln n_{e}}{d \psi}\left(\frac{\partial J}{\partial \psi}-\frac{k_{\psi}}{k_{\alpha}} \frac{\partial J}{\partial \alpha}\right)
$$

is negative. In a quasi-isodynamic stellarator $(\partial J / \partial \alpha=0)$ with the maximum- $J$ property (which in practice can be approached at high $\beta$ ), we conclude that $P_{e}>0$, so that the electrons exert a stabilising influence on any electrostatic modes as long as $0<\eta_{e}<2 / 3$. One would thus not expect any conventional, density-gradient driven, collisionless TEMs in this situation. In practice, it is not possible to achieve either of the conditions $\partial J / \partial \alpha=0$ and $\partial J / \partial \psi<0$ for all trapped orbits, but this idealised limit is approached in well-optimised stellarator designs such as W7-X.

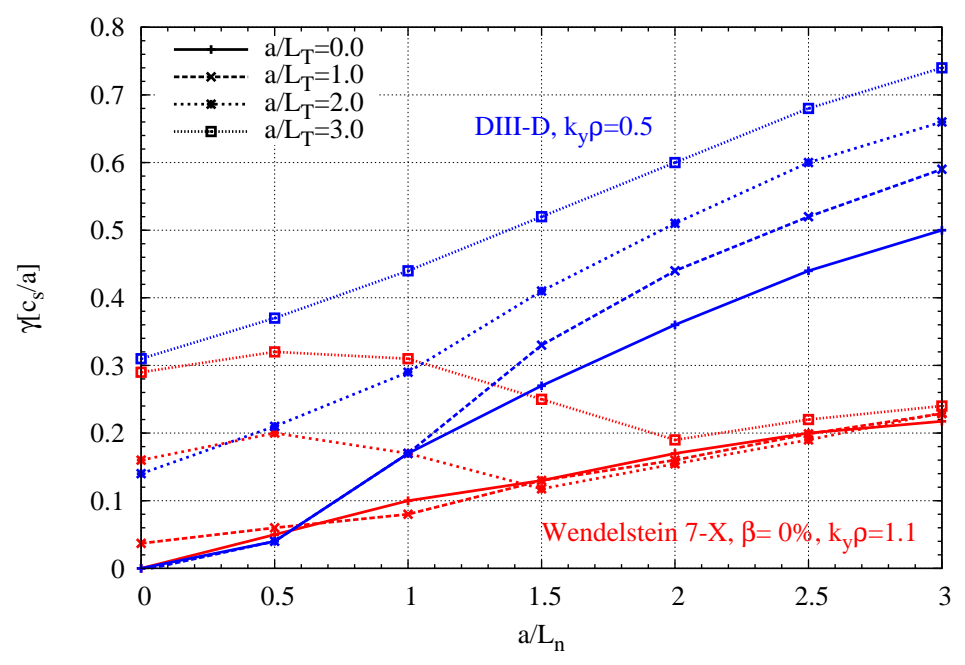

Figure 7: Growth rate of the fastest growing mode on the flux surface $s=1 / 2$ in W7-X and DIII-D, calculated with GENE, as functions of the density and temperature gradient scale lengths normalised to the minor radius. The electron and ion temperatures, and their gradients, are taken to be equal. The geometry is that of a flux tube, passing through the outer midplane in the bean-shaped cross section of W7-X.

To test these analytical predictions, a series of systematic flux-tube simulations have been carried out comparing TEMs in a typical tokamak (DIII-D), NCSX, W7$\mathrm{X}$ and a more quasi-isodynamic stellarator configuration, using the GENE code. In 
the tokamaks, all flux tubes on the same flux surface are equivalent, but this is not the case in stellarator geometry. The simulations presented here were done for the flux tube that crosses the horizontal midplane in the bean-shaped cross section. This flux tube is expected to be the most unstable one, because of the large unfavourable curvature in this cross section, and this expectation was confirmed by comparison with simulations in the other up-down symmetric flux tube of the stellarators in question. More importantly, the results show that TEMs are more stable the farther the regions of bad curvature and magnetic trapping are separated from each other [12], and that the energy flow from the electrons is indeed as predicted by the theory. Figure 7 shows a comparison between the fastest growing modes in DIII-D and W7-X. In these simulations, the electron and ion temperatures, and their gradients, were taken to be equal, so there is potential for both ITG modes and TEMs. At zero density gradient, where ITG modes dominate, the growth rates are similar, but a finite density gradient is far less destabilising in the stellarator. Not only is the growth rate lower, but the most unstable mode has a shorter perpendicular wavelength. The traditional quasilinear estimate for the diffusion coefficient $D \sim \gamma / k_{\perp}^{2}$ thus suggests much lower transport in the stellarator when the density gradient is large. Strictly speaking, the best estimate is obtained by maximising $\gamma / k_{\perp}^{2}$ over $k_{\perp}$ rather than by first maximising $\gamma\left(k_{\perp}\right)$ and then dividing by $k_{\perp}^{2}$, but these estimates tend, of course, to be correlated to one another.

\section{Turbulent transport}

This expectation is supported by the first nonlinear GENE simulations with kinetic electrons in flux-tube geometry. These results have not yet been verified by any other code, but show interesting differences between the turbulent transport in the two devices. Figure 8 shows the evolution of the heat flux normalised to the gyro-Bohm value

$$
Q_{g b}=\frac{\rho_{i}^{2} v_{T i} p_{i}}{a^{2}}
$$

where $p_{i}=n_{i} T_{i}$ is the ion pressure, $v_{T i}=\left(T_{i} / m_{i}\right)^{1 / 2}$ the thermal speed and $\rho_{i}=v_{T i} / \Omega_{i}$ the gyroradius of the ions. The simulations are done with normalised ion temperature and density gradients of $a / L_{T_{i}}=3$ and $a / L_{n}=1$, respectively, and without an electron temperature gradient. When normalised to the gyro-Bohm value in this way, the heat 
flux density is an order of magnitude smaller in W7-X than in DIII-D. The difference is even larger if the density gradient is increased and the ion temperature gradient is suppressed, so that ITG modes are eliminated, as in Fig. 9, where $a / L_{T_{e}}=1$ and $a / L_{n}=2$.

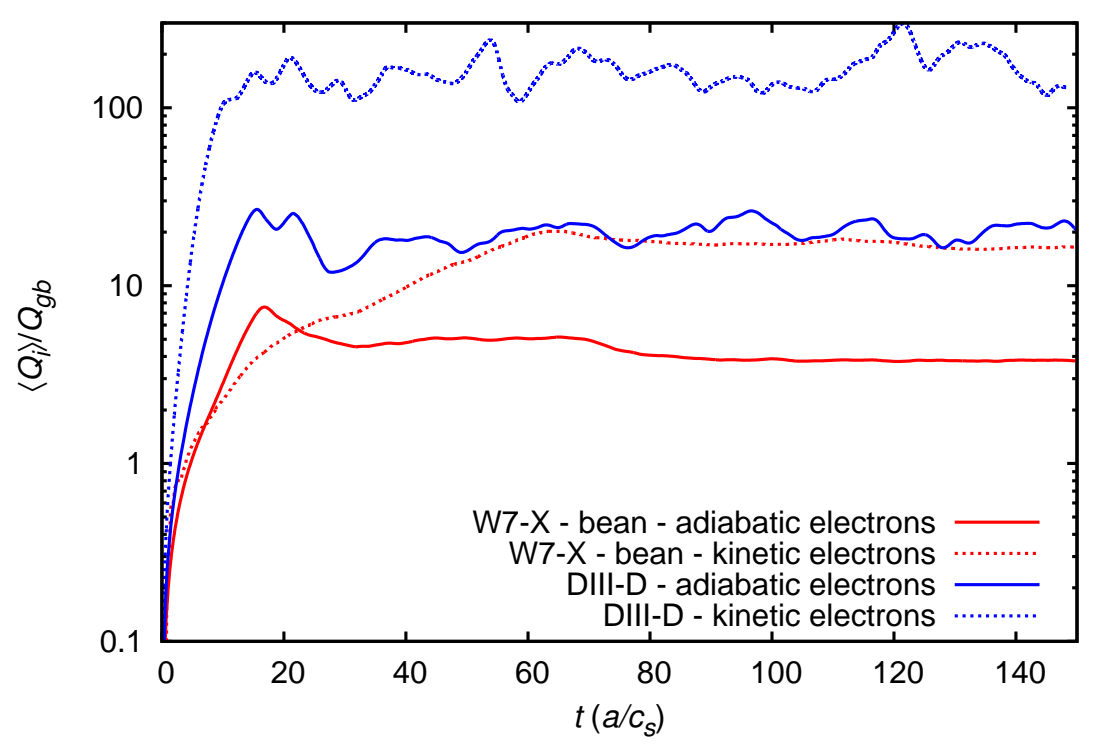

Figure 8: Turbulent heat flux in flux-tube simulations of DIII-D and W7-X. The gradients are $a / L_{n}=1, a / L_{T_{i}}=3$, and $a / L_{T_{e}}=0$.

It is however too early to draw any conclusions about the overall turbulent transport and confinement properties of stellarators compared with tokamaks. Firstly, these simulations ignore collisions, the radial electric field and electromagnetic effects, and are only possible in flux-tube geometry at the moment. Furthermore, they are performed far above the stability threshold and cannot be considered sufficiently realistic. Secondly, it must be borne in mind that W7-X has a larger surface-to-volume ratio than DIII-D, which adversely affects the confinement. In a fusion experiment, what counts in practice is the heating power $P$ and magnetic-field energy $W$ required to achieve some desired plasma parameters. When comparing two devices, it is thus natural to scale them to the same volume and field strength to make $W$ the same [1]. In a global simulation, one could then apply the same heating power to the two devices, run the simulation for several confinement times, and measure the resulting fusion triple prod- 
uct $n T \tau$. This would be a fair way to compare the confinement of two fusion devices with different geometry.

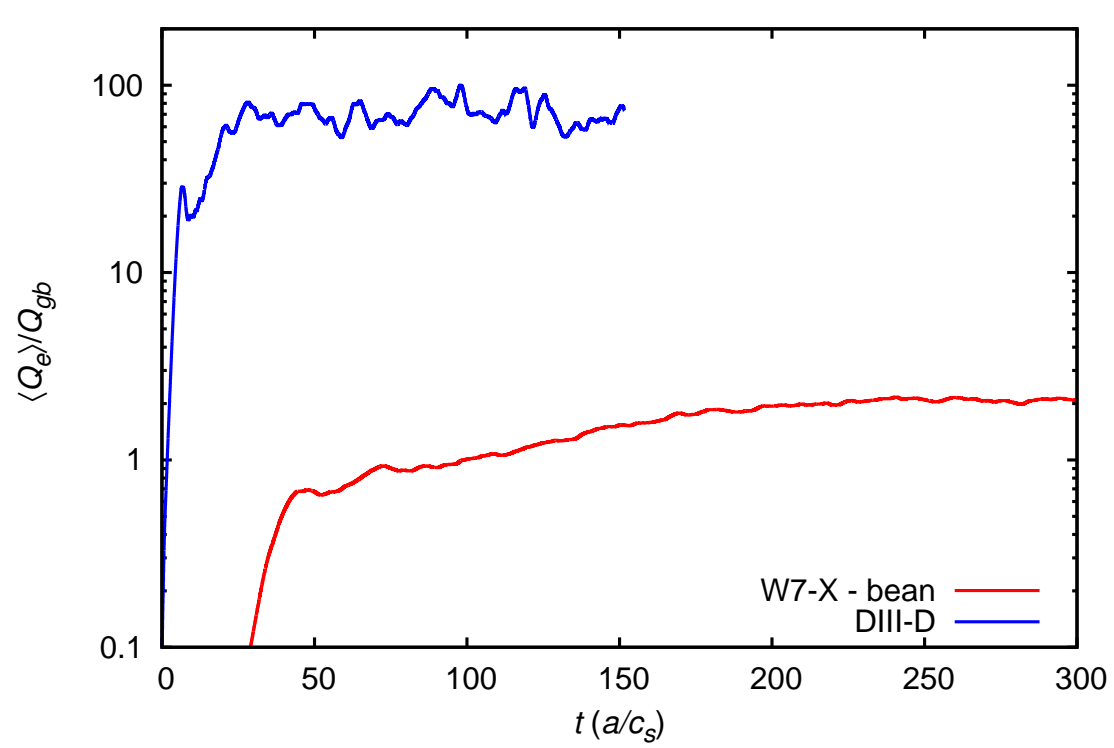

Figure 9: Turbulent heat flux in flux-tube simulations of DIII-D and W7-X. The gradients are $a / L_{n}=2, a / L_{T_{i}}=0$, and $a / L_{T_{e}}=1$. The DIII-D simulation saturated earlier and was therefore interrupted.

It is not as straightforward to compare two devices on the basis of a local turbulence simulation results, such as those of Figs. 8 and 9, but one can proceed as follows. The total power crossing the flux surface in question is equal to the surface integral

$$
P=\int \frac{\mathbf{Q} \cdot \nabla r}{|\nabla r|} d S=A\langle\mathbf{Q} \cdot \nabla r\rangle,
$$

where angular brackets denote the conventional flux-surface average and $A=V^{\prime}(r)$ is the radial derivative of the volume enclosed by the surface. $A$ thus has the dimension of area, and $P$ is equal to

$$
P=\rho_{i}^{2} v_{T i} p_{i} \frac{A \hat{Q}}{a^{2}},
$$

where $\hat{Q}=\langle\mathbf{Q} \cdot \nabla r\rangle / Q_{g b}$ is the heat flux density normalised to the gyro-Bohm value. If the local plasma parameters are assumed to be the same in the two devices, it is clear that the severity of the turbulent losses is characterised not by $\hat{Q}$ but by $A \hat{Q} / a^{2}$. The dimensionless factor $A / a^{2}$ is about five times larger larger in W7-X than in DIII-D, so 
$\hat{Q}$ needs to be at least five times smaller to ensure the same confinement. This is indeed the case in Figs. 8 and 9. In these particular, very idealised, simulations, the turbulent transport thus appears to be smaller in W7-X. It should perhaps be remarked that the heat flux plotted in Figs. 8 and 9 is not the flux-surface-averaged one, but that across the flux tube used in the simulations, which is the one that intersects the outer midplane in the bean-shaped cross section of W7-X. Since this flux tube is particularly unstable linearly, one would expect that it overestimates the transport.

\section{Conclusions}

Both ITG modes and TEMs are present in gyrokinetic stellarator simulations, but exhibit interesting differences from their tokamak counterparts. The "bad" magnetic curvature is very unevenly distributed over each flux surface in a stellarator such as W7-X, and the flux surfaces are tightly spaced in some regions but far removed from one another elsewhere. The result is that the density and potential fluctuations associated with both the linear eigenmodes and saturated turbulence form thin structures along the magnetic field, whose narrow width appears to affect the transport. However, the growth rates of ITG modes in W7-X, both with Boltzmann-distributed and kinetic electrons, appear to be comparable to those in a typical tokamak at similar values of $a / L_{T}$, and the difference is even less if the tokamak is chosen to have a similar connection length between good and bad curvature [13].

Trapped-electron-modes display even greater differences, particularly in stellarators where the maximum- $J$ condition is approximately satisfied and most trapped orbits therefore enjoy good average curvature. In such configurations, these electrons precess poloidally in the direction opposite to that in which drift waves propagate, and therefore cannot cause resonant destabilisation of such waves. (This is otherwise how the collisionless TEM arises.) Moreover, as predicted by the analytical theory, the flow of electrostatic energy is such that the electrons are mostly stabilising and, indeed, no traditional TEMs are observed in the simulations. Other instabilities are present, but these are driven by the ions rather than the electrons. When the density gradient is large, the maximum growth rate is found to be significantly lower than in typical tokamaks, and the corresponding wavelength shorter. 
Although it remains to be seen how large the corresponding effect is on the transport in systematic studies of saturated turbulence, the first nonlinear simulations indicate that the turbulent transport is significantly lower in W7-X than in a typical tokamak, when measured in gyro-Bohm units. This difference is partly offset by the greater surface-to-volume ratio of $\mathrm{W} 7-\mathrm{X}$. The simulations neglect a range of potentially important effects and cannot be considered to be sufficiently realistic, but may nevertheless be indicative of different turbulence properties in stellarators and tokamaks.

\section{Appendix: Free-energy balance}

In the main text, we used the electrostatic energy balance to draw conclusions about stability. Specifically, the electrons were considered to be stabilizing or destabilizing depending on whether or not they perform work on the fluctuations. However, it should also be mentioned that stability can also be analyzed in a different way, using the entropy (by some authors called the "free energy") budget of the system [19, 20, 21].

This is done by multiplying the gyrokinetic equation (1) by $g_{a}^{*} /\left(f_{a 0} B\right)$ and integrating over velocity space and along the field line. The first term,

$$
\left\{\frac{i g_{a}^{*} v_{\|} \nabla g_{a}}{f_{a 0}}\right\}=i \int_{0}^{\infty} \frac{\pi v^{3} d v}{f_{a 0}} \int_{0}^{1 / B_{\min }} d \lambda \sum_{\sigma, j} \sigma \int g_{a}^{*} \nabla_{\|} g_{a} d l
$$

where $\sigma=v_{\|} /\left|v_{\|}\right|$and the sum over $j$ runs over all trapping wells along the field line [11], then becomes purely real, and the imaginary part of the other terms imply

$$
\gamma\left\{\frac{\left|g_{a}\right|^{2}}{f_{a 0}}\right\}=\operatorname{Im} \frac{e_{a}}{T_{a}}\left\{J_{0} \phi g_{a}^{*}\left(\omega-\omega_{* a}^{T}\right)\right\} .
$$

Multiplying this equation by $T_{a}$, summing over all species, and using the quasineutrality condition,

$$
\sum_{a} \frac{n_{a} e_{a}^{2}}{T_{a}} \phi=\sum_{a} e_{a} \int g_{a} J_{0} \mathrm{~d}^{3} v
$$

gives the following expression for the growth rate,

$$
\gamma=-\operatorname{Im} \sum_{a} e_{a}\left\{J_{0} \phi g_{a}^{*} \omega_{* a}^{T}\right\} / \sum_{a}\left\{\frac{T_{a}\left|g_{a}\right|^{2}}{f_{a 0}}-\frac{e_{a}^{2} f_{a 0}|\phi|^{2}}{T_{a}}\right\},
$$

where the denominator is positive definite since it can be written in terms of the total perturbed distribution function as

$$
\sum_{a}\left\{\frac{T_{a}\left|g_{a}\right|^{2}}{f_{a 0}}-\frac{e_{a}^{2} f_{a 0}|\phi|^{2}}{T_{a}}\right\}=\sum_{a} T_{a}\left\{\frac{\left|f_{a 1}\right|^{2}}{f_{a 0}}\right\} .
$$


Hence and from the expression for the entropy density,

$$
-f_{a} \ln f_{a}=-f_{a 0} \ln f_{a 0}-\left(1+\ln f_{a 0}\right) f_{a 1}-\frac{f_{a 1}^{2}}{2 f_{a 0}}+O\left(f_{a 1}^{3}\right),
$$

it is clear that Eq. (3) is related to the entropy balance (or, perhaps more accurately, the Helmholtz free energy) of the system. It is tempting to use this equation to make statements about the stabilizing or destabilizing effect of various particle species. If the quantity

$$
\tilde{Q}_{a}(\omega)=-\operatorname{Im}\left\{e_{a} J_{0} \phi g_{a}^{*} \omega_{* a}^{T}\right\},
$$

is positive for a particular species, then the latter apparently gives off free energy to the fluctuations and acts destabilizing in terms of the free-energy budget.

However, for our present purposes this treatment of the stability problem is not entirely straightforward. One difficulty is that a certain species may be stabilizing in terms of electrostatic energy but destabilizing in terms of free energy. Another, and more serious, problem is that the sum of the quantities $\tilde{Q}_{a}(\omega)$, i.e., the numerator in Eq. (3) does not, in general, vanish at the point of marginal stability. This circumstance can be proved generally, but is perhaps more easily seen from a simple example. Let us consider an instability with $\omega \ll k_{\|} v_{T e}$ in a plasma with $\eta_{i}=0$, so that

$$
\tilde{Q}_{i}(\omega)=-\operatorname{Im}\left\{e \omega_{* i} J_{0} \phi g_{e}^{*}\right\}
$$

because of quasineutrality. Substituting Eq. (2) in the expression

$$
\tilde{Q}_{i}(\omega)+\tilde{Q}_{e}(\omega)=-\operatorname{Im}\left\{e\left(\omega_{* i}-\omega_{* e}^{T}\right) \phi g_{e}^{*}\right\}
$$

and using Eq. (3) then gives

$$
\gamma \sum_{a} T_{a}\left\{\frac{\left|f_{a 1}\right|^{2}}{2 f_{a 0}}\right\}=\frac{\gamma e^{2}}{T_{e}}\left\{\frac{\left(\bar{\omega}_{d e}-\omega_{* e}^{T}\right)\left(\omega_{* i}-\omega_{* e}^{T}\right)}{\left(\omega_{r}-\bar{\omega}_{d e}\right)^{2}+\gamma^{2}}|\bar{\phi}|^{2} f_{e 0}\right\},
$$

where we have written $\omega=\omega_{r}+i \gamma$. The right-hand side does not vanish as $\gamma \rightarrow 0$, which implies that the rate of entropy production is finite at the marginal stability point (if $|\phi|$ is finite), because the integral on the left of the equation diverges in this limit.

\section{Acknowledgement}

This work has been carried out within the framework of the EUROfusion Consortium, and has received funding from the European Unions Horizon 2020 research and inno- 
vation programme under grant agreement number 633053. The views and opinions expressed herein do not necessarily reflect those of the European Commission. 


\section{References}

[1] TURKIN, Y., BEIDLER, C. D., MAASSBERG, H., MURAKAMI, S., TRIBALDOS, V., WAKASA, A., Phys. Plasmas 18 (2011) 022505.

[2] HIRSCH, M. et al, Plasma Phys. Control. Fusion 50 (2008) 053001.

[3] KORNILOV, V., KLEIBER, R., HATZKY, R., VILlARD, L., JOST, G., Phys. Plasmas 11 (2004) 3196.

[4] JENKO, F., DORLAnd, W., KOTSCHENREUTHER, M., ROGERS, B.N., Phys. Plasmas 7 (2000) 1904.

[5] XAnthopoulos, P., Merz, F., GÖRler, JenkO, F., Phys. Rev. Lett. 99 (2007) 035002.

[6] XANThopoulos, P., MYNICK, H., HELANDER, P., TURKIN, YU., PLUNK, G.G., JENKO, F., GÖRLER, T., TOLD, D., BIRD, T.M., PROLL, J.H.E., Phys. Rev. Lett. 113 (2014) 155001.

[7] KENDL, A., Plasma Phys. Control. Fusion 43 (2001) 1559.

[8] WATAnabe, T.-H., SUGAma, H., FERRAndO-MARGalet, S., Phys. Rev. Lett. 100 (2008) 195002.

[9] BAumgaertel, J. A., HAMmetT, G. W., MiKKELSEN, D. R., NUNAMi, M., XANTHOPOUlOS, P., Phys. Plasmas 19 (2012) 122306.

[10] HELANDER, P., BIRD, T.M., DREVlAK, M., FENG, Y., HATZKY, R., JENKO, F., KLEIBER, R., PROLL, J.H.E., TURKIN, YU., XANTHOPOULOS, P., Plasma, Phys. Control. Fusion 54 (2012) 124009.

[11] Helander, P., PROLL, J.H.E., PLUNK, G.G., Phys. Plasmas 20 (2013) 122505 .

[12] PROLL, J.H.E., HElAnder, P., XANThopoulos, P., Phys. Plasmas 20 (2013) 122506 .

[13] PlunK, G.G., Helander, P., XANThopoulos, P., CONNOR, J.W., Phys. Plasmas 21 (2014) 032112. 
[14] GEIGER, J., BEIDLER, C.D., FENG, Y., MAASSBERG, H., MARSHCHENKO, N.B., TURKIN, Y., Plasma Phys. Control. Fusion 57 (2014) 014004,

[15] MCMILLAN, B. F.,LAPILLONNE, X., BRUNNER, S., VILLARD, L., JOLLIET, S., BOtTino, A., GÖRLDER, T., and JENKO, F. Phys. Rev. Lett. 105 (2010) 155001.

[16] HELANDER, P., Rep. Progr. Phys. 77 (2014) 087001.

[17] ROSEnBluth, M.N., Phys. Fluids 11 (1968) 869.

[18] PROLL, J.H.E., HELAnder, P., CONnOR, J.W., PlunK, G.G., Phys. Rev. Lett. 108 (2012) 245002.

[19] SugAma, H., OKAmoto, W., HORTOn, W., WAKATAni, M., Phys. Plasmas 3 (1996) 2379.

[20] HALlATSCHEK, K., Phys. Rev. Lett. 93 (2004) 065001.

[21] SCHEKOCHIHIN, A.A., COWLEY, S.C., DORLAND, W., HAMMETT, G.W., Howes, G.G., QuAetaert, E., TAtSunO, T., Ap. J. (Suppl. Series) 182 (2009) 310. 